\title{
A!
}

This is an electronic reprint of the original article.

This reprint may differ from the original in pagination and typographic detail.

Kopnin, N.B.; Volovik, G.E.

\section{Rotating vortex core: An instrument for detecting core excitations}

Published in:

Physical Review B

DOI:

10.1103/PhysRevB.57.8526

Published: 01/01/1998

Document Version

Publisher's PDF, also known as Version of record

Please cite the original version:

Kopnin, N. B., \& Volovik, G. E. (1998). Rotating vortex core: An instrument for detecting core excitations.

Physical Review B, 57(14), 8526-8531. https://doi.org/10.1103/PhysRevB.57.8526

This material is protected by copyright and other intellectual property rights, and duplication or sale of all or part of any of the repository collections is not permitted, except that material may be duplicated by you for your research use or educational purposes in electronic or print form. You must obtain permission for any other use. Electronic or print copies may not be offered, whether for sale or otherwise to anyone who is not an authorised user. 


\title{
Rotating vortex core: An instrument for detecting core excitations
}

\author{
N. B. Kopnin \\ Landau Institute for Theoretical Physics, 117334 Moscow, Russia; \\ Laboratoire de Physique des Solides, Université Paris-Sud, Bâtiment 510, 91405, Orsay, France; \\ and Helsinki University of Technology, Low Temperature Laboratory, P.O. Box 2200, FIN-02015 HUT, Finland \\ G. E. Volovik \\ Helsinki University of Technology, Low Temperature Laboratory, P.O. Box 2200, FIN-02015 HUT, Finland \\ and Landau Institute for Theoretical Physics, 117334 Moscow, Russia
}

(Received 11 June 1997; revised manuscript received 19 December 1997)

\begin{abstract}
Effects of fermion zero modes (bound states in a vortex core) on the rotational dynamics of vortices with spontaneously broken axisymmetry are considered. The results are compared with the Helsinki NMR experiments where the vortex cores were driven to a fast rotation and torsional oscillations by an NMR rf field. We predict a resonance NMR absorption on localized states at the external frequency comparable with the interlevel distance which is similar to the cyclotron Landau damping. The resonances can resolve the localized levels in vortex cores. For a pure rotation of the core, the effect depends on the relative signs of the vortex winding and of the core rotation; thus it is sensitive to the direction of rotation of the container. The similarity with fermion zero modes on fundamental strings, which simulate the thermodynamics of black holes, is discussed. [S0163-1829(98)05214-X]
\end{abstract}

\section{INTRODUCTION}

Low-frequency dynamics of vortices in Fermi superfluids and superconductors is determined by low-energy excitations. If the energy spectrum of fermionic quasiparticles in the bulk superfluid or superconducting system has a finite gap, the relevant low-energy quasiparticles occur only in vortex cores. The spectrum of fermions bound to the vortex core has an anomalous branch, which crosses zero as a function of the angular momentum. It was first found by Caroli, de Gennes, and Matricon ${ }^{1}$ for axisymmetric vortices in $s$-wave superconductors. Effects of these quasiparticles on vortex dynamics were considered in a number of papers (see, for example, Refs. 2 and 3); some of the effects have the same origin as the chiral anomaly in quantum field theory. ${ }^{4}$ Other phenomena appear if pairing occurs in a state with a different order-parameter symmetry. For example, a peculiar dynamics has been recently predicted for vortices in $d$-wave superconductors: Due to the localized excitations which are concentrated near the gap nodes a finite dissipation exists even in the limit of a very long relaxation time. This happens due to the Landau damping on zero-sound collective modes in the one-dimensional (1D) Fermi liquid of the localized states. ${ }^{5}$ The particular importance of bound states near the gap nodes in $d$-wave superconductors has been already demonstrated for the density of states: ${ }^{6}$ a single-vortex density of states was predicted to exhibit a singular $1 / E$ energy dependence resulting in a temperature independent vortex contribution to the specific heat.

In the present paper, we consider effects of bound states on dynamics of nonaxisymmetric vortices which may exist in superfluids with unconventional pairing. In particular, we consider vortices whose nonaxisymmetric cores can rotate with respect to the heat bath. This case is distinct from dynamics of (generally nonaxisymmetric) vortices in $d$-wave superconductors where, however, the orientation of vortex cores is fixed by the crystal lattice, and only a translational motion is possible. Such nonaxisymmetric vortices exist, for example, in superfluid ${ }^{3} \mathrm{He}-\mathrm{B}$ where the superfluid environment and the heat bath are homogeneous and isotropic, while the vortex core exhibits spontaneous anisotropy in the crossectional plane. This spontaneous breaking of the continuous $\mathrm{U}(1)$ symmetry (axial symmetry) of a single vortex core occurs below the vortex-core transition ${ }^{7,8}$ and is similar to the breaking of the electromagnetic U(1) symmetry in the core of a cosmic string (the so-called superconducting string). ${ }^{9}$

In ${ }^{3} \mathrm{He}-\mathrm{B}$ vortex experiments, the nonaxisymmetric vortex cores were driven to fast rotational oscillations plus rotation with a constant velocity under an applied rf field. ${ }^{10}$ In the present paper, we resolve the existing controversy concerning estimations of the effect of the core anisotropy on vortex dynamics. ${ }^{10} \mathrm{We}$ find that the main effects come from the fermion zero modes in the nonaxisymmetric vortex core. In particular, they lead to resonances which should be observed at lower temperatures when the external frequency of the core oscillations $\omega$ is a multiple of the distance between the energy quantum levels in the core. Such resonances were first predicted in Ref. 11 for the translational motion of the axisymmetric vortices in superconductors, but they were never observed due to a difficulty of exciting translational vortex oscillations with the required frequency.

We also consider the case when the core rotates around its axis with a constant angular velocity $\Omega$. The frictional torque has an asymmetry with respect to the relative signs of the vortex winding number and the sense of core rotation; it thus can be detected by changing the direction of one of the rotations. When $\Omega$ becomes equal to the interlevel spacing gapless fermions appear; this has a close similarity to a $d$-wave superconductor.

Finally, we discuss our results in connection with the 
similarity between the fermion zero modes in cores of vortices in condensed matter and fermion zero modes in cores of topological objects in cosmology (cosmic strings, walls, monopoles, etc.). This similarity allows us to model the process of electroweak baryogenesis in the core of a cosmic string using the superfluid ${ }^{3} \mathrm{He}-\mathrm{B} .{ }^{4} \mathrm{We}$ consider here another aspect of vortex dynamics related to fermion zero modes: namely, the possibility to simulate the event horizon and Hawking radiation by a rotating vortex core. In this connection, the fermion zero modes in a vortex core act exactly as zero modes in fundamental strings in string description of black holes.

\section{HPD DRIVEN CORE OSCILLATIONS}

In Ref. 10 the asymmetric cores of vortices were coherently driven into a rotational and oscillatory motion using a special and unique dynamical state with the coherently precessing magnetization, known as the homogeneously precessing domain (HPD). This phase-coherent precession is a magnetic counterpart of a superfluid coherent state and has the similar off-diagonal long-range order, thus representing the magnetic superfluidity. The principles of HPD can be found in the review. ${ }^{12}$ The coherent precession has been used to study different properties of superfluid ${ }^{3} \mathrm{He}-\mathrm{B}$. In particular, (i) vortices with an asymmetric core ${ }^{10}$ (analogs of the Witten superconducting strings ${ }^{9}$ ) and (ii) vortices formed at termination lines of solitons ${ }^{13}$ (analog of strings as terminations of walls in cosmology ${ }^{14}$ ), have been detected using HPD.

In the HPD, the magnetization precesses with the rf frequency $\omega_{\mathrm{rf}}$. The core is driven into motion due to the dipole torque according to the following equation: ${ }^{10}$

$$
f_{\text {fr }} \dot{\gamma}=-T_{D} \sin 2\left(\omega_{\mathrm{rf}} t-\gamma\right)+T_{R} \sin 2 \gamma .
$$

Here $\gamma$ is the angle of the core anisotropy director $\mathbf{d}$ in the plane transverse to the vortex axis, $\mathbf{d}=\hat{x} \cos \gamma+\hat{y} \sin \gamma$ [see Fig. 1(a)]; the dipole torque $T_{D}$ and the friction parameter $f_{\text {fr }}$ are nonzero if the core is anisotropic. The restoring torque $T_{R}$ appears in a tilted magnetic field which determines a preferred orientation for the vortex anisotropy director. Under the condition of the experiment, the friction torque $\sim \omega_{\mathrm{rf}} f_{\mathrm{fr}}$ is large compared with the driving dipole torque $T_{D}$. As a result, the core performs small and fast oscillations superimposed on a slower drift $\beta(t)$ :

$$
\gamma(t)=\gamma_{\omega} \cos 2\left(\omega_{\mathrm{rf}} t-\beta\right)+\beta .
$$

The effective drift velocity is nonzero for a comparatively small restoring torque $T_{R}<T_{D}^{2} / 2 \omega_{\mathrm{rf}} f_{\mathrm{fr}}$. From Eq. (1) it follows that for zero restoring torque, the drift angular velocity is $\Omega \equiv \dot{\beta}=T_{D}^{2} / 2 f_{\mathrm{fr}}^{2} \omega_{\mathrm{rf}}$, i.e., it is smaller than the HPD precession frequency. The amplitude of core oscillations is $\gamma_{\omega}=T_{D} / 2 f_{\text {fr }} \omega_{\mathrm{rf}}$.

In principle, the core can perform a pure rotational motion, too. This happens either for small friction $2 \omega_{\mathrm{rf}} f_{\mathrm{fr}}<T_{D}$ when $\Omega=\omega_{\text {rf }}$, or if the core is twisted by the NMR rf field: after the external drive is switched off the core starts to untwist through a free motion. ${ }^{10} \mathrm{We}$ consider here both pure rotation and pure oscillations.

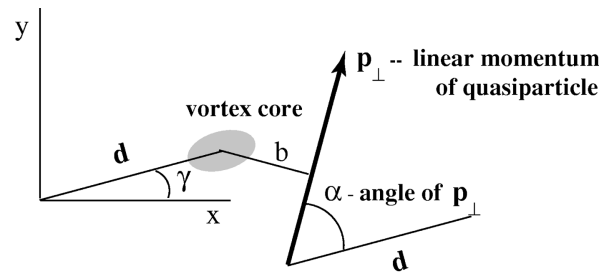

(a)

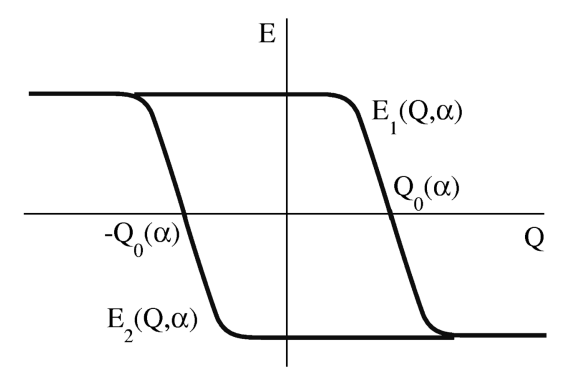

(b)

FIG. 1. (a) Trajectory of the quasiparticle near the asymmetric vortex core. Angle $\gamma$ shows the orientation of the core anisotropy vector $\mathbf{d}$; angle $\alpha$ shows the direction of the transverse linear momentum $\mathbf{p}_{\perp}$ of the quasiparticle with respect to $\mathbf{d}$; and $b$ is the impact parameter. (b) Quantization of the fast motion along the trajectory gives two branches of low-energy spectrum $E_{1,2}(b, \alpha)$ in terms of the rest (slow) variables $b$ and $\alpha$. Instead of the impact parameter it is better to use the continuous angular momentum $Q=b\left|\mathbf{p}_{\perp}\right|$. As a function of $Q$ the spectrum $E_{1,2}(Q, \alpha)$ crosses zero at two antisymmetric values of $Q$, which depend on $\alpha$ : $Q= \pm Q_{0}(\alpha)$. At the next stage the true quantum mechanical levels are obtained by quantization of slow motion using the BohrSommerfeld rule for the canonically conjugated variables $Q$ and $\alpha$ : $\oint d \alpha Q(\alpha, E)=2 \pi n \hbar$. This gives the equidistant levels $E=n E_{0}$.

The dominating contribution to the friction comes from kinetics of fermions in the rotating core which is thus the primary object of our investigation. We find that Eq. (1) has a very limited applicability since it does not contain the proper frequency dependence. We derive the correct vortex dynamics which also includes the resonance absorption, i.e., the Landau damping on the "zero-sound-like" modes in the system of localized fermions in the vortex core.

\section{EFFECT OF FERMIONIC ZERO MODES ON CORE DYNAMICS}

Quasiparticles bound to the vortex. The low-energy spectrum of Caroli-de Gennes-Matricon quasiparticles around a vortex contains an anomalous branch of fermion zero modes. Excitations on this branch are characterized by two canonically conjugated variables, the angle $\alpha$ of the transverse linear momentum $\mathbf{p}_{\perp}$ of the quasiparticle, say, with respect to the core anisotropy direction d [see Fig. 1(a)], and the angular momentum $Q$. For superconductors with the coherence length $\xi$ much larger than the inverse Fermi momentum $p_{F} \xi \gg 1$ the electron wavelength is short compared with the core size, and the quasiclassical approximation is relevant. In the quasiclassical approximation, the variables $\alpha$ and $Q$ commute and the spectrum has the form

$$
E(Q, \alpha)=-\omega_{0}(\alpha)\left[Q-Q_{0}(\alpha)\right] .
$$


The quasiclassical angular momentum $Q$ is a continuous variable; thus the anomalous branch crosses zero as a function of $Q$ at $Q=Q_{0}(\alpha)$. In particular, one has $Q_{0}=0$ when the vortex-core structure is completely symmetric. $Q_{0}$ becomes finite if parity is violated. This was found by Schopoh ${ }^{15}$ for a nonaxisymmetric vortex in ${ }^{3} \mathrm{He}-\mathrm{B}$; two anomalous branches, which cross zero at two antisymmetric values $Q= \pm Q_{0}(\alpha)$, are schematically shown on Fig. 1(b). The magnitude of $Q_{0}(\alpha)$ in continuous vortices in ${ }^{3} \mathrm{He}-\mathrm{A}$ is determined by the core size of the vortex $\left|Q_{0}(\alpha)\right| \sim p_{F} R_{\text {core }} \cdot{ }^{16,17}$ For ${ }^{3} \mathrm{He}-\mathrm{B}$, where the core radius is of order of coherence length $\xi$, this estimation gives a large value of $Q_{0} \sim p_{F} \xi$, in accordance with the unpublished calculations by Schopohl for the nonaxisymmetric vortex with broken parity, the so-called $v$ vortex. ${ }^{15}$

The kinetic equation for the distribution function $f(t, \alpha, Q)$ of fermions on the anomalous branch for a system characterized by canonically conjugated variables has a conventional form: ${ }^{18}$

$$
\frac{\partial f}{\partial t}+\frac{\partial f}{\partial \alpha} \frac{\partial E}{\partial Q}-\frac{\partial E}{\partial \alpha} \frac{\partial f}{\partial Q}=-\frac{f-f_{0}}{\tau} .
$$

Here $\partial E / \partial Q=-\omega_{0}(\alpha) ; f_{0}$ is the equilibrium Fermi distribution function.

If the vortex core rotates the quasiparticle energy depends on time according to $E(Q, \alpha, t)=E[Q, \alpha-\gamma(t)]$. After transformation $\alpha-\gamma(t) \rightarrow \alpha$ one obtains the following kinetic equation:

$$
\frac{\partial f}{\partial t}-\left(\omega_{0}+\dot{\gamma}\right) \frac{\partial f}{\partial \alpha}-\frac{\partial E}{\partial \alpha} \frac{\partial f}{\partial Q}=-\frac{f-f_{0}}{\tau} .
$$

One can write

$$
f_{1}=f-f_{0}=\left(d f_{0} / d E\right) \chi .
$$

If $\dot{\gamma}=\dot{\gamma}_{\omega} e^{-i \omega t}=-i \omega \gamma_{\omega} e^{-i \omega t}$ is a perturbation with a frequency $\omega=2 \omega_{\mathrm{rf}}$, one has

$$
\left(-i \omega+\frac{1}{\tau}\right) \chi-\omega_{0} \frac{\partial \chi}{\partial \alpha}-\frac{\partial E}{\partial \alpha} \frac{\partial \chi}{\partial Q}=\dot{\gamma}_{\omega} \frac{\partial E}{\partial \alpha} .
$$

The friction torque on a rotating core from fermions on the bound states in the core is

$$
\begin{aligned}
T_{f}(\omega) & =-\int \frac{d p_{z}}{2 \pi} \int \frac{d \alpha d Q}{2 \pi} \frac{\partial Q}{\partial t} \chi \frac{\partial f_{0}}{\partial E} \\
& =\int \frac{d p_{z}}{2 \pi} \int \frac{d \alpha d Q}{2 \pi} \frac{\partial E}{\partial \alpha} \frac{d f_{0}}{d E} \chi .
\end{aligned}
$$

The eigenstates or zero-sound-like collective modes in the Fermi liquid of localized states are obtained as solutions of the homogeneous equation, Eq. (7), without the driving term on the right-hand side (RHS):

$$
\chi_{0}(\alpha, Q, \omega)=\Lambda[E(\alpha, Q)] e^{F(\alpha, \omega)},
$$

$$
F(\alpha, \omega)=\int_{0}^{\alpha} d \alpha^{\prime} g\left(\alpha^{\prime}, \omega\right), \quad g(\alpha, \omega)=\frac{-i \omega+1 / \tau}{\omega_{0}(\alpha)},
$$

where $\Lambda(E)$ is an arbitrary function of $E$. The eigenvalues are obtained from the requirement of periodicity $\chi(\alpha, Q)=\chi(\alpha+2 \pi, Q)$ :

$$
\int_{0}^{2 \pi} d \alpha g(\alpha, \omega)=2 \pi n i,
$$

where $n$ is an integer. In the collisionless regime, this gives eigenfrequencies of the collective modes:

$$
\omega=n E_{0}, \frac{1}{E_{0}}=\int_{0}^{2 \pi} \frac{d \alpha}{2 \pi} \frac{1}{\omega_{0}(\alpha)} \equiv\left\langle\frac{1}{\omega_{0}}\right\rangle .
$$

Here $E_{0}\left(p_{z}\right)$ is an exact minigap as distinct from the quasiclassical minigap $\omega_{0}\left(p_{z}\right)$ (compare with Ref. 5). The Landau damping on these discrete frequencies occurs even in the collisionless regime. It is completely analogous to the Landau cyclotron damping. Let us consider this in more detail.

The general solution of Eq. (7) can be found from the following consideration. Since both the energy $E(\alpha, Q)=-\omega_{0}\left(Q-Q_{0}\right)$ and the RHS of Eq. (7) are binomials of $Q$, a general solution can also be represented as a binomial in $Q$, and thus as a binomial in $E$ :

$$
\chi(\alpha, Q, \omega)=[a(\alpha, \omega) E(\alpha, Q)+b(\alpha, \omega)] e^{F(\alpha, \omega)} .
$$

The functions $a$ and $b$ satisfy the equations

$$
\frac{\partial a}{\partial \alpha}=-\frac{\dot{\gamma}_{\omega}}{\omega_{0}^{2}} \frac{\partial \omega_{0}}{\partial \alpha} e^{-F(\alpha, \omega)}, \frac{\partial b}{\partial \alpha}=-\dot{\gamma}_{\omega} \frac{\partial Q_{0}}{\partial \alpha} e^{-F(\alpha, \omega)} .
$$

At small $T$ the linear-in- $E$ term in Eq. (13) is small and thus only the equation for $b(\alpha, \omega)$ is important. The solution for $b$ consistent with the periodicity of $\chi$ gives for $E \rightarrow 0$

$$
\begin{aligned}
\chi(\alpha, \omega)= & \dot{\gamma}_{\omega} e^{F(\alpha, \omega)}\left[\int_{0}^{\alpha} d \alpha^{\prime} \frac{\partial Q_{0}}{\partial \alpha^{\prime}} e^{-F\left(\alpha^{\prime}, \omega\right)}\right. \\
& \left.+\frac{1}{e^{-F(\omega)}-1} \int_{0}^{2 \pi} d \alpha \frac{\partial Q_{0}}{\partial \alpha} e^{-F(\alpha, \omega)}\right],
\end{aligned}
$$

where $F(\omega) \equiv F(2 \pi, \omega)$.

Landau cyclotron damping. In the collisionless limit and close to a pole at $\omega=n E_{0}$ one has

$$
\begin{aligned}
\chi\left(\alpha, \omega \approx n E_{0}\right)= & -\frac{\dot{\gamma}_{\omega}}{2 \pi i} \frac{E_{0}}{\omega-n E_{0}+i / \tau} \\
& \times e^{F\left(\alpha, n E_{0}\right)} \int_{0}^{2 \pi} d \alpha \frac{\partial Q_{0}}{\partial \alpha} e^{-F\left(\alpha, n E_{0}\right)} .
\end{aligned}
$$

The torque on the core is 


$$
T_{f}(\omega)=-\dot{\gamma}_{\omega} \int \frac{d p_{z}}{2 \pi} G\left(\omega, p_{z}\right) \equiv-\dot{\gamma} f_{\mathrm{fr}}(\omega)
$$

Close to the resonance one has the response

$$
\begin{aligned}
G\left(\omega, p_{z}\right)= & \frac{i E_{0}}{\omega-n E_{0}+i / \tau} \times\left\langle\frac{\partial Q_{0}}{\partial \alpha} e^{F\left(\alpha, n E_{0}\right)}\right\rangle \\
& \times\left\langle\frac{\partial Q_{0}}{\partial \alpha} e^{-F\left(\alpha, n E_{0}\right)}\right\rangle .
\end{aligned}
$$

Let us consider the resonance absorption in the simple model in which $\omega_{0}$ does not depend on $\alpha$ while the core anisotropy is represented only by the function $Q_{0}(\alpha)$. This model is close to what was found by Schopohl. ${ }^{15}$ In the simple model of constant $\omega_{0}$, one has

$$
G\left(\omega, p_{z}\right)=\frac{i E_{0} n^{2}\left|Q_{0 n}\right|^{2}}{\omega-n E_{0}+i / \tau}, \quad Q_{0 n}=\int \frac{d \alpha}{2 \pi} Q_{0}(\alpha) e^{i n \alpha},
$$

where $Q_{0 n}$ is the $n$th harmonic of the $Q_{0}(\alpha)$.

The $p_{z}$ dispersion of the exact minigap $E_{0}\left(p_{z}\right)$ results in formation of an absorption band. ${ }^{11}$ If the frequency $\omega$ falls within the band, the poles in $G\left(\omega, p_{z}\right)$ give rise to the Landau damping even in the completely collisionless limit: the friction coefficient is

$$
\operatorname{Re} f_{\mathrm{fr}}(\omega)=\sum_{n>0} E_{0}\left(p_{z n}\right) n\left|Q_{0 n}\left(p_{z n}\right)\right|^{2}\left[\left|\frac{d E_{0}}{d p_{z}}\right|_{p_{z n}}\right]^{-1},
$$

where $p_{z n}$ is the momentum for which the resonance condition $n E_{0}\left(p_{z n}\right)=\omega$ takes place.

NMR absorption: Collisionless regime. Equation (1) is modified due to the frequency dispersion of the response function:

$$
f_{\text {fr }}(\omega) \dot{\gamma}_{\omega}=-T_{D},
$$

which gives the NMR resonant absorption

$$
P=\frac{1}{2} \operatorname{Re} f_{\mathrm{fr}}(\omega)\left|\dot{\gamma}_{\omega}\right|^{2}=\frac{1}{2} T_{D}^{2} \operatorname{Re} f_{\mathrm{fr}}^{-1}(\omega) .
$$

In the collisionless regime, Eq. (19) can be used. The resonant absorption starts with a sharp minimum in the background absorption. Indeed, if $\omega$ is at the edge of the band $n E_{0}\left(p_{z}\right)$ where $d E_{0} / d p_{z}=0$ the absorption proportional to $\left[\operatorname{Re} f_{\text {fr }}(\omega)\right] /\left|f_{\text {fr }}(\omega)\right|^{2}$ decreases sharply due to an infinitely large real part of $f_{\text {fr }}(\omega)$. It goes exactly to zero in the ideal collisionless limit. Observation of these "van Hove" zeroes would resolve the minigap in NMR experiments.

NMR absorption: Hydrodynamic limit. In the hydrodynamic limit $\omega_{0} \tau \ll 1$ we have $F(\omega) \gg 1$, and the first term in Eq. (15) dominates. It results in a local expression

$$
\chi=\dot{\gamma}_{\omega} \frac{\partial Q_{0}}{\partial \alpha} \frac{\omega_{0}}{-i \omega+1 / \tau} .
$$

The friction parameter which enters the NMR absorption in Eq. (21) becomes

$$
\operatorname{Re} f_{\mathrm{fr}}^{-1}(\omega)=\frac{1}{\tau}\left[\frac{p_{F}}{\pi}\left(\omega_{0}\left(\frac{\partial Q_{0}}{\partial \alpha}\right)^{2}\right\rangle_{F}\right]^{-1} .
$$

where $\langle\cdots\rangle_{F}$ is the average over the Fermi surface.

The experiment of Ref. 10 has been carried out in the temperature range $0.48 T_{c}<T<0.6 T_{c}$ while, according to Ref. 4, the hydrodynamic regime $\tau \omega_{0}<1$ occurs only above $T \sim 0.6 T_{c}$. Let us compare, however, the theory and experiment at $T=0.6 T_{c}$. The experimental value of the friction parameter in Eq. (1), $f_{\mathrm{fr}} \sim 5 \times 10^{-18} \mathrm{erg} \mathrm{s} / \mathrm{cm}$ at $T=0.6 T_{c},{ }^{10}$ can be obtained if one takes $\sqrt{\left\langle\left(\partial Q_{0} / \partial \alpha\right)^{2}\right\rangle} \sim 10$. This large magnitude of the derivative $\partial Q_{0} / \partial \alpha$ is not inconsistent with the Schopohl result: $Q_{0} \sim p_{F} \xi$. However, since $T$ is not small in the experiment, the $T^{2}$ term which results from the omitted energy-dependent terms in Eq. (15) can be important and can give a comparable contribution to $f_{\text {fr }}$. The temperature is to be decreased to resolve the value of $Q_{0}$.

\section{UNIFORM CORE ROTATION}

If the vortex core rotates with a constant angular velocity $\Omega$ the asymptotic solution is time independent and obeys the equation

$$
\frac{1}{\tau} \chi-\left(\omega_{0}+\Omega\right) \frac{\partial \chi}{\partial \alpha}-\frac{\partial E}{\partial \alpha} \frac{\partial \chi}{\partial Q}=\Omega \frac{\partial E}{\partial \alpha}
$$

The case of a slow rotation is equivalent to Eq. (22) with $\omega=0$ and $\dot{\gamma}=\Omega$. In the hydrodynamic limit, we thus have Eq. (23).

The case of a fast rotation $\Omega \sim \omega_{0}$ can be considered as a linear response only in the limit of a small anisotropy $\partial \omega_{0} / \partial \alpha \ll \omega_{0}$ and $\partial Q_{0} / \partial \alpha \ll Q_{0}$. Using the perturbation theory in the anisotropy parameters, we can solve the kinetic equations for both high and small relaxation rate. Using the ansatz of Eq. (13), we find the periodic solution

$$
\chi=\int_{0}^{2 \pi} d \alpha^{\prime}\left[\frac{\Theta\left(\alpha-\alpha^{\prime}\right)}{e^{\Phi}-1}+\frac{\Theta\left(\alpha^{\prime}-\alpha\right)}{1-e^{-\Phi}}\right] b\left(\alpha^{\prime}\right) e^{\Phi\left(\alpha-\alpha^{\prime}\right)},
$$

where

$$
\begin{gathered}
b(\alpha)=\frac{\Omega \omega_{0}}{\omega_{0}+\Omega} \frac{\partial Q_{0}}{\partial \alpha}, \\
\Phi(\alpha)=\frac{\alpha}{\tau\left(\omega_{0}+\Omega\right)}, \quad \Phi \equiv \Phi(2 \pi) .
\end{gathered}
$$

For $\quad T \rightarrow 0 \quad$ when $\quad d f_{0} / d E=-\delta\left(Q-Q_{0}\right) / \omega_{0} \quad$ and $d E / d \alpha=\omega_{0} d Q_{0} / d \alpha$, one obtains the response function

$$
\begin{aligned}
G(0)= & \frac{\omega_{0}}{\omega_{0}+\Omega} \int_{0}^{2 \pi} \frac{d \alpha d \alpha^{\prime}}{2 \pi}\left[\frac{\Theta\left(\alpha-\alpha^{\prime}\right)}{e^{\Phi}-1}\right. \\
& \left.+\frac{\Theta\left(\alpha^{\prime}-\alpha\right)}{1-e^{-\Phi}}\right] \frac{\partial Q_{0}}{\partial \alpha} \frac{\partial Q_{0}}{\partial \alpha^{\prime}} e^{\Phi\left(\alpha-\alpha^{\prime}\right)} .
\end{aligned}
$$


Resonance. Equation (28) essentially depends on the sense of the rotation of the vessel, which determines the relative signs of $\omega_{0}$ and $\Omega$. The most interesting situation occurs when $\omega_{0}$ and $\Omega$ have opposite signs. In this case the resonance absorption should occur when $-\Omega$ is close to $\omega_{0}$. In the hydrodynamic regime $\left|\omega_{0}+\Omega\right| \ll 1 / \tau$ the frictional torque coincides with $\mathrm{Eq}$. (23). In the intermediate regime $\left|\omega_{0}+\Omega\right| \gg 1 / \tau \gg\left|\omega_{0}-\langle\omega\rangle\right|$ one obtains

$$
f_{\mathrm{fr}}=\frac{p_{F}}{\pi}\left\langle\frac{\omega_{0}}{\left(\omega_{0}+\Omega\right)^{2}} \frac{\left\langle Q_{0}^{2}\right\rangle-\left\langle Q_{0}\right\rangle^{2}}{\tau}\right\rangle_{F} .
$$

Using Eq. (29) and Eq. (23), one can approximate the resonance absorption by

$$
f_{\mathrm{fr}}=p_{F}\left\langle\omega_{0} \delta\left(\omega_{0}+\Omega\right)\left[\left\langle Q_{0}^{2}\right\rangle-\left\langle Q_{0}\right\rangle^{2}\right]\right\rangle_{F} .
$$

If, for the proper sense of rotation, the function $\omega_{0}\left(p_{z}\right)+\Omega$ crosses zero at some $p_{z 0}$, the friction remains finite even in the limit $\tau \gg 1 / \omega_{0}$ (however, $\tau$ has to be small compared to the inverse dispersion):

$$
\begin{aligned}
f_{\mathrm{fr}} & =\int \frac{d p_{z}}{2} \omega_{0} \delta\left(\omega_{0}+\Omega\right)\left[\left\langle Q_{0}^{2}\right\rangle-\left\langle Q_{0}\right\rangle^{2}\right] \\
& =\frac{\omega_{0}}{\left|d \omega_{0} / d p_{z}\right|_{p_{z 0}}}\left[\left\langle Q_{0}^{2}\right\rangle-\left\langle Q_{0}\right\rangle^{2}\right] .
\end{aligned}
$$

In the simple-core model where $\omega_{0}$ is independent of $\alpha$ and $Q_{0}$ has only one harmonic,

$$
Q_{0}(\alpha)= \pm g \cos \alpha
$$

the solution is

$$
\chi=A \sin \alpha+B \cos \alpha, \quad A=-\frac{\omega_{0} \Omega \tau g}{1+\left(\omega_{0}+\Omega\right)^{2} \tau^{2}} .
$$

The factor $B$ is not important since it does not contribute to the friction torque. The friction torque has a resonance behavior:

$$
G\left(p_{z}\right)=\frac{g^{2}}{2} \frac{\omega_{0} \tau}{1+\left(\omega_{0}+\Omega\right)^{2} \tau^{2}}
$$

in agreement with both Eq. (29) for collisionless regime and Eq. (23) for the hydrodynamic regime.

\section{DISCUSSION}

The interesting problems to be discussed in the future are related to the resonance behavior, which occurs when the edge of the minigap band $\omega_{0}(\alpha)$ as a function of $\alpha$ crosses the angular velocity $\Omega$ of the uniform rotation. If the bottom of the band is chosen at $\alpha=0$ (and also at $\alpha=\pi$ due to symmetry of the core) one has near the bottom

$$
\omega_{0}(\alpha)+\Omega \approx \omega_{0}(0)+\Omega+\frac{1}{2} \beta \alpha^{2}, \quad \beta=d^{2} \omega_{0}(0) / d \alpha^{2} .
$$

At the moment when $-\Omega$ touches the bottom $\omega_{0}(0)$, the shifted minigap $\omega_{0}(\alpha)+\Omega$ has nodes of a second order. The same situation occurs for nonrotating vortices in the $d$-wave superconductors. The higher-order gap nodes have serious consequences for the vortex thermodynamics ${ }^{19,6}$ and dynamics. ${ }^{5}$ In particular, the dissipation occurs even in the superclean limit.

Let us start with a small positive $\omega_{0}(0)+\Omega$ and then continue it analytically to a negative value. We are interested in the Bose-factor in Eq. (25) in the collisionless limit $\tau=\infty$ with a finite frequency $\omega$. Substituting $1 / \tau$ with $-i \omega$, we find for $0<\omega_{0}(0)+\Omega \ll \omega_{0}(0)$

$$
\begin{aligned}
\Phi & \approx-2 i \omega \int_{-\pi / 2 \omega_{0}(0)+\Omega+(1 / 2) \beta \alpha^{2}}^{\pi / 2} \frac{d \alpha}{} \\
& \approx-\frac{2 \pi i \omega \sqrt{2}}{\sqrt{\beta\left[\omega_{0}(0)+\Omega\right]}} .
\end{aligned}
$$

Here we took into account that the integral is concentrated in the vicinity of $\alpha=0$ and also in the vicinity of $\alpha=\pi$. The analytical continuation of Eq. (35) to a small negative $\omega_{0}(0)+\Omega$ gives a real quantity

$$
\Phi \approx-\frac{2 \pi \omega \sqrt{2}}{\sqrt{\beta\left|\Omega+\omega_{0}(0)\right|}}=-4 \pi \frac{\omega}{\left|\partial \omega_{0} / \partial \alpha\right|_{\omega_{0}(\alpha)=-\Omega}} .
$$

The Bose factor in Eq. (25) thus corresponds to an equilibrium distribution with an effective temperature determined by the derivative of the minigap $\omega_{0}(\alpha)$ at the point where $\omega_{0}(\alpha)=-\Omega$ :

$$
\frac{1}{e^{\omega / T_{\mathrm{eff}}-1}}, \quad T_{\mathrm{eff}}=\frac{1}{4 \pi}\left|\left(\frac{\partial \omega_{0}}{\partial \alpha}\right)_{\omega_{0}(\alpha)=-\Omega}\right| .
$$

This has a close relation with the event horizon problem. ${ }^{20}$ Recently there was a profound progress in understanding of the Bekenstein-Hawking ${ }^{21,22}$ black hole thermodynamics using the fundamental strings (see recent review paper by Horowitz ${ }^{23}$ ). The statistical origin of the black hole thermodynamics is related to fermion and boson zero modes of strings. Excitations having the lowest energy are confined to a circle and are chiral: they are, say, right moving along the circle. Fermions localized in the core of a quantized vortex in a superconductor or in a Fermi superfluid of the type of superfluid ${ }^{3} \mathrm{He}$ have similar properties. ${ }^{1}$ The model of a vortex core where fermions live on a closed line around the vortex axis in real space was discussed in Ref. 24. The lowest energy branch (the fermion zero mode in the vortex) represents 1+1 massless fermions, which are left moving (right moving) around the axis of the vortex, if the vortex has a positive (negative) winding number. The axial anomaly caused by the fermion zero modes during motion of vortices (strings) in ${ }^{3} \mathrm{He}$ and superconductors ${ }^{25}$ has experimentally observable consequences for the vortex dynamics. ${ }^{4}$

If the canonically conjugated variables on the circle, i.e., the angle $\alpha$ and the angular momentum $Q$, are represented as the coordinate $x=\alpha$ and the momentum $p=Q$, the spectrum of fermion zero modes of the type of Eq. (3) describes a massless relativistic particle left moving along the circle: 


$$
E(p, x)=-c(x)[p-A(x)] .
$$

Here $c(x)=\omega_{0}(x)$ plays the part of the speed of light, and $Q_{0}(\alpha)=A(x)$ is the vector potential. This spectrum is universal, though the details, i.e., the functions $c(x)=\omega_{0}(\alpha)$ and $A(x)=Q_{0}(\alpha)$, depend on a particular vortex structure in a particular superfluid or superconductor.

The gas of these $1+1$ massless chiral fermions has the required statistical properties. For example, if we consider the axisymmetric core, the quasiparticle energy spectrum in terms of the angular momentum quantum number $Q$ is $E=-\omega_{0} Q$. For such spectrum the number of quantum states with the fixed total angular momentum $\mathcal{Q}$ is ${ }^{26}$

$$
e^{S}, S=2 \pi \sqrt{\mathcal{Q} / 12} .
$$

The same happens also for the nonaxisymmetric vortex, since its energy spectrum is also linear in quantum number, $E=n E_{0}$ according to Eq. (12). This means that if the vortex has the nonzero momentum $\mathcal{Q}$ (this happens, for example, if two identical vortices rotate about their center of mass or if the nonaxisymmetric core rotates) the fermionic entropy $S$ of the core appears to be nonzero even at $T=0$. This violation of the "Nernst theorem" is similar to that in the black hole. ${ }^{23,26}$ Also the discrete spectrum of the radiation by the rotating core, with frequencies which are integral multiple of $E_{0}$ in Eq. (12), is similar to that by the quantum black hole. ${ }^{27}$

In the classical limit the rotation of the nonaxisymmetric vortex can lead to the "event horizon." This happens when
$|\Omega|>\omega_{0}(0)$ and the "speed of light" $c(x)=\omega_{0}(\alpha)+\Omega$ crosses zero at some values of $x$. The analogue of the Hawking temperature is to be played by the spatial derivative of the speed of light at horizon $T_{\text {Hawking }}=(1 / 2 \pi) \mid(d c /$ $d x)_{\text {hor }}|=|\left(d \omega_{0} / d \alpha\right)_{\omega_{0}(\alpha)=-\Omega} \mid$. This is two times larger than the effective temperature in Eq. (37) probably because of two "black holes" in the core (close to $\alpha=0$ and close to $\alpha=\pi$ ).

To summarize, we predict a resonant absorption on localized levels in an asymmetric vortex core performing rotational oscillations which are similar to the cyclotron Landau damping. For a pure rotation of the core around its axis with a constant angular velocity $\Omega$, the frictional torque has an asymmetry with respect to the relative signs of the vortex winding number and the sense of core rotation.

\section{ACKNOWLEDGMENTS}

G.E.V. thanks Ted Jacobson for the collaboration on the horizon problem. This work was supported by the Russian Foundation for Fundamental Research Grant No. 96-0216072 and by the program "Statistical Physics" of the Ministry of Science of Russia. N.B.K. also acknowledges the support by the Swiss National Foundation cooperation Grant No. 7SUP J048531 and by the INTAS Grant No. 96-0610. G.E.V. acknowledges the support by the European Science Foundation.
${ }^{1}$ C. Caroli, P. G. de Gennes, and J. Matricon, Phys. Lett. 9, 307 (1964)

${ }^{2}$ N. B. Kopnin and V. E. Kravtsov, Pis'ma Zh. Eksp. Teor. Fiz. 23, 631 (1976) [ JETP Lett. 23, 578 (1976)].

${ }^{3}$ N. B. Kopnin and A. V. Lopatin, Phys. Rev. B 51, 15291 (1995).

${ }^{4}$ T. D. C. Bevan, A. J. Manninen, J. B. Cook, J. R. Hook, H. E. Hall, T. Vachaspati, and G. E. Volovik, Nature (London) 386, 689 (1997).

${ }^{5}$ N. B. Kopnin and G. E. Volovik, Phys. Rev. Lett. 79, 1377 (1997).

${ }^{6}$ N. B. Kopnin and G. E. Volovik, Pis'ma Zh. Eksp. Teor. Fiz. 64, 641 (1996) [ JETP Lett. 64, 690 (1996)].

${ }^{7}$ E. V. Thuneberg, Phys. Rev. Lett. 56, 359 (1986).

${ }^{8}$ M. M. Salomaa and G. E. Volovik, Phys. Rev. Lett. 56, 363 (1986).

${ }^{9}$ E. Witten, Nucl. Phys. B249, 557 (1985).

${ }^{10}$ Y. Kondo, J. S. Korhonen, M. Krusius, V. V. Dmitriev, Yu. M. Mukharsky, E. B. Sonin, and G. E. Volovik, Phys. Rev. Lett. 67, 81 (1991).

${ }^{11}$ N. B. Kopnin, Pis'ma Zh. Eksp. Teor. Fiz. 27, 417 (1978) [ JETP Lett. 27, 390 (1978)].

${ }^{12}$ Yu. M. Bunkov, in Progress in Low Temperature Physics, edited by W. Halperin (Elsevier, New York, 1995), Vol. 14, pp. 69158.
${ }^{13}$ Y. Kondo, J. S. Korhonen, M. Krusius, V. V. Dmitriev, E. V. Thuneberg, and G. E. Volovik, Phys. Rev. Lett. 68, 3331 (1992).

${ }^{14}$ A. Vilenkin and E. P. S. Shellard, Cosmic Strings and Other Topological Defects (Cambridge University Press, Cambridge, 1994); M. B. Hindmarsh and T. W. B. Kibble, Rep. Prog. Phys. 58, 477 (1995).

${ }^{15}$ N. Schopohl (unpublished).

${ }^{16}$ N. B. Kopnin, Physica B 210, 267 (1995).

${ }^{17}$ G. E. Volovik, Pis'ma Zh. Eksp. Teor. Fiz. 61, 935 (1995) [ JETP Lett. 61, 958 (1995)].

${ }^{18}$ M. Stone, Phys. Rev. B 54, 13222 (1996).

${ }^{19}$ G. E. Volovik, Pis'ma Zh. Eksp. Teor. Fiz. 58, 457 (1993) [ JETP Lett. 58, 469 (1993)].

${ }^{20}$ T. Jacobson and G. E. Volovik, cond-mat/9801308 (unpublished).

${ }^{21}$ J. D. Bekenstein, Phys. Rev. D 7, 2333 (1973).

${ }^{22}$ S. Hawking, Nature (London) 248, 30 (1974).

${ }^{23}$ G. T. Horowitz, gr-qc/9704072 (unpublished).

${ }^{24}$ G. E. Volovik, cond-mat/9709159 (unpublished).

${ }^{25}$ N. B. Kopnin, G. E. Volovik, and Ü. Parts, Europhys. Lett. 32, 651 (1995).

${ }^{26}$ R. M. Wald, Phys. Rev. D 56, 6467 (1997).

${ }^{27}$ J. D. Bekenstein, gr-qc/9710076 (unpublished). 